Proceedings of the Online Conference "Applications of Physics in Mechanical and Material Engineering"

\title{
Numerical Calculations of VGs Influence on Aerodynamic Characteristics of Airfoil
}

\author{
R. Gnatowska*, K. Gajewska and R. Kańtoch
}

Częstochowa University of Technology, 42-201 Częstochowa, Poland

Doi: $10.12693 /$ APhysPolA.139.586

*e-mail: gnatowska@imc.pcz.pl

\begin{abstract}
Flow separation on aerodynamically shaped bodies is always an unfavorable phenomenon responsible for substantial energy losses. Passive vortex generators (VGs) are widely used as a simple and effective tool to delay or suppress separation on an airfoil. Despite many studies and practical applications of this flow control method, there are still many scientific problems related to VGs application that need to be solved. For example, the optimal VGs geometry and their positioning to have the best aerodynamic effect have not always been studied in enough detail. The present work describes a computational fluid dynamic analysis of VGs application on an airfoil NLF-MOD22B with a flap model of the Extra EA-400. A case of an airfoil with a strongly deflected rear flap inducing a strong detachment was considered. A vortex generator was located at the lower side of the airfoil near the local trailing edge upstream of the flap. The analysis of the results, especially the pressure distribution and skin friction coefficient on the airfoil indicates that VGs are capable of delaying flow separation over the flap. It was observed that both the lift and drag coefficient depends on the applied shape of the VGs. The research indicates that the selection of the optimal geometry of the disturbing element is very difficult: although its application increases the lifting force of the entire profile, on the other hand, it also influences the drag force and as a result, the maximum lift-drag ratio $\left(C_{\mathrm{L}} / C_{\mathrm{D}}\right)$ is similar to the case without VGs. An optimistic result, however, is the observation that a beneficial effect is noticed when analyzing the flop itself.
\end{abstract}

topics: airfoil, numerical calculations, aerodynamics, flow control

\section{Introduction}

The airfoils have a wide range of applications from aerospace to unmanned aerial vehicles and wind turbine blades. It should be noted that such profiles operate under various conditions and speed ranges, from slow gliders to supersonic military aircraft. Due to their wide application, they constitute a serious challenge for engineers in terms of the required airfoil performance [1].

An important aspect in the design of aircraft wings is the aerodynamics of high payload. This is especially important in two basic phases of an airplane flight, i.e., the take-off, and the landing. In these phases of flight, an increased lifting force is required, which can be achieved by using an airfoil with a leading and/or trailing edge flap. Today, many flap systems still use obsolete, complex solutions from the early 1940s. However, more and more often new, simpler, and above all, cheaper solutions are sought. Due to the increase in the computing power of computers, flow tests are more and more often carried out around devices with a high load capacity, using new solutions, such as numerical methods CFD (computational fluid dynamics) [2].

The available literature offers a variety of different approaches allowing for flow control and some of them are up to now widely applied in practice. In addition to VG, methods such as plasma flow control, micro-passes, micro-toys, blowing and suction, synthetic nozzles, and flexible walls are increasingly used in the design or optimization of airfoils to improve their aerodynamic performance. One interesting flow control method based on the physics of the boundary layer is the use of a wall, with a streamwise waviness topology [3]. Barlas [4] and Johnson [5] compared different types of flow control methods, while Lin [6] and Wang [7] found VG to be one of the most effective devices for improving blade aerodynamics. Lin [8] investigated the subsurface VG boundary layer, where they reduce flow separation in a manner comparable to their larger conventional counterparts, without increasing drag. These generators, by turning in opposite directions, increased the lift on the leash by $10 \%$, while the rear blade lobe reduced drag by $50 \%$. This is the result of a delay in separation from about $45 \%$ of the airfoil chord to at least $85 \%$ [2].

The main objective of this work is to study the flow around the high lift NLF-MOD22B airfoil, with a single slotted trailing edge flap system. For flapped airfoil systems, the flow aerodynamics is complex, which is due to the mutual interaction 
of the main profile and the flap. Particularly complex conditions exist when there is a strong deflection of the flap that induced flow separation. To achieve a better aerodynamic performance of the airfoil, we decided to apply a vortex generator (VG) located at the lower side of the airfoil near the local trailing edge and to analyze their effectiveness using numerical methods.

\section{Methodology}

The profile of NLF-MOD22B airfoil with a flap was shown in Fig. 1. The chord of the tested panel is $0.6 \mathrm{~m}$, and the span is $1.25 \mathrm{~m}$. The size of the flap is $30 \%$ of the chord length. The tests were carried out for selected parameters of the airfoil setting, i.e., for a zero inflow angle $\alpha=0^{\circ}$ and the flap set at the angle $\delta=55^{\circ}$. The vortex generator (VG) was located at the lower side of the airfoil near the local trailing edge to delay separation over the flap. Two shapes of VG were tested: triangle (case $\mathrm{T}$ ) and rectangle (case $\mathrm{R}$ ), both with the height of $6 \mathrm{~mm}$. The position and height of $\mathrm{VG}$ were determined based on the results presented by Jansen [2].

Numerical simulations for $\operatorname{Re}=2.0 \times 10^{6}$ were made using the Ansys Fluent 2020 R2 program. The validation of the results was performed using the wind tunnel data obtained from the work of Jansen [2], where the influence of VG, in the shape of a triangle, in two positions was examined. As a first reference case, calculations were performed for the airfoil without the vortex generator.

The solution domain is the C-shaped geometry, as shown in Fig. 2a, and the resulted unstructured meshing consists of triangle elements which were done by ANSYS Meshing (Fig. 2b). The mesh in the wall area was designed so that the $y_{1}^{+}$parameter was less than 5. The dimension of the computational domain used for the $2 \mathrm{D}$ calculations is $15 \mathrm{~m}$ in the $x$ direction and $12 \mathrm{~m}$ in the $y$ direction and with a distance of 15 blade chord $c$ behind the airfoil.

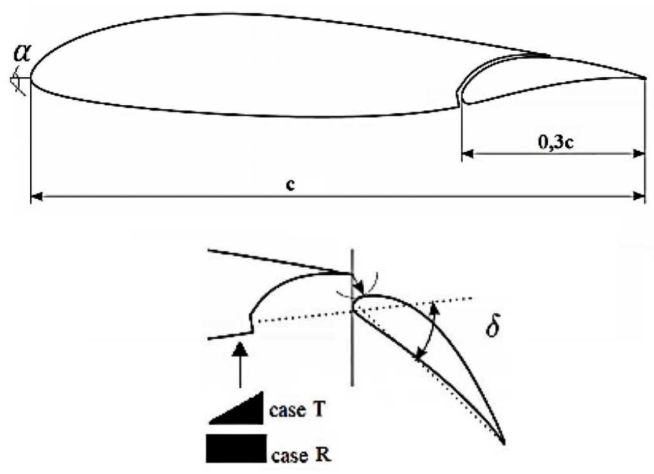

Fig. 1. Scheme of NLF MOD22B airfoil with VG location, where: $c$ - the chord, $\delta$ - the flap angle, $\alpha$ - the inlet angle.

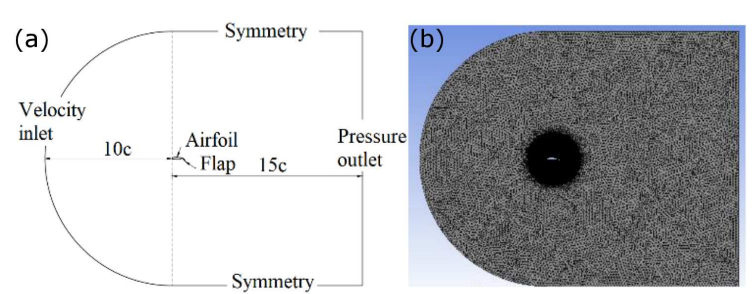

Fig. 2. Computational domain with boundary conditions (a) and the computational mesh among the whole domain (b).

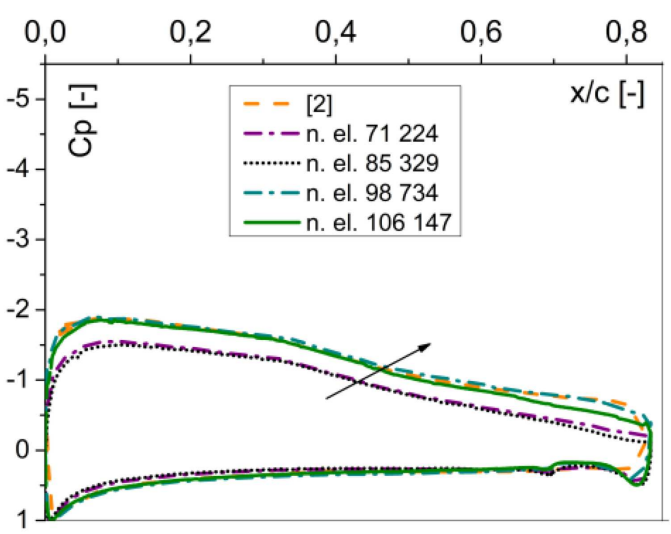

Fig. 3. Variation of pressure coefficient $C_{\mathrm{p}}$ for various numbers of elements.

The research was carried out using the ReynoldsAveraged Navier-Stokes (RANS) method with the use of the $k-\omega$ turbulence model in the SST version. The applied boundary conditions at the inlet were: velocity $50 \mathrm{~m} / \mathrm{s}$, ambient pressure $101325 \mathrm{~Pa}$, and inlet turbulence intensity $0.1 \%$. At the outlet a condition was set: pressure outlet.

A series of simulations were conducted for mesh independency tests, for reference configuration, i.e., airfoil without VGs. A different number of meshes were created by diving circular and rectangular sections with a different number of elements. Figure 3 depicts the variation of the pressure coefficient for different numbers of mesh elements plotted with reference data from Jansen [2]. Based on the grid independence tests, it is concluded that mesh with 98734 elements seems to be fine for the present study to obtain the optimum results. Also, the obtained lift coefficient $C_{\mathrm{L}}$ has a value close to the result given by Jansen [2].

\section{Results and discussion}

Figure 4 presents pressure distributions $C_{\mathrm{p}}$ on the airfoil and the flap without and with both VG models. The upper curves represent pressure coefficient distribution on the upper surface of the airfoil with the flap, while the lower curves correspond to the lower surface. 
TABLE I

VGs influence on aerodynamic characteristics of the airfoil.

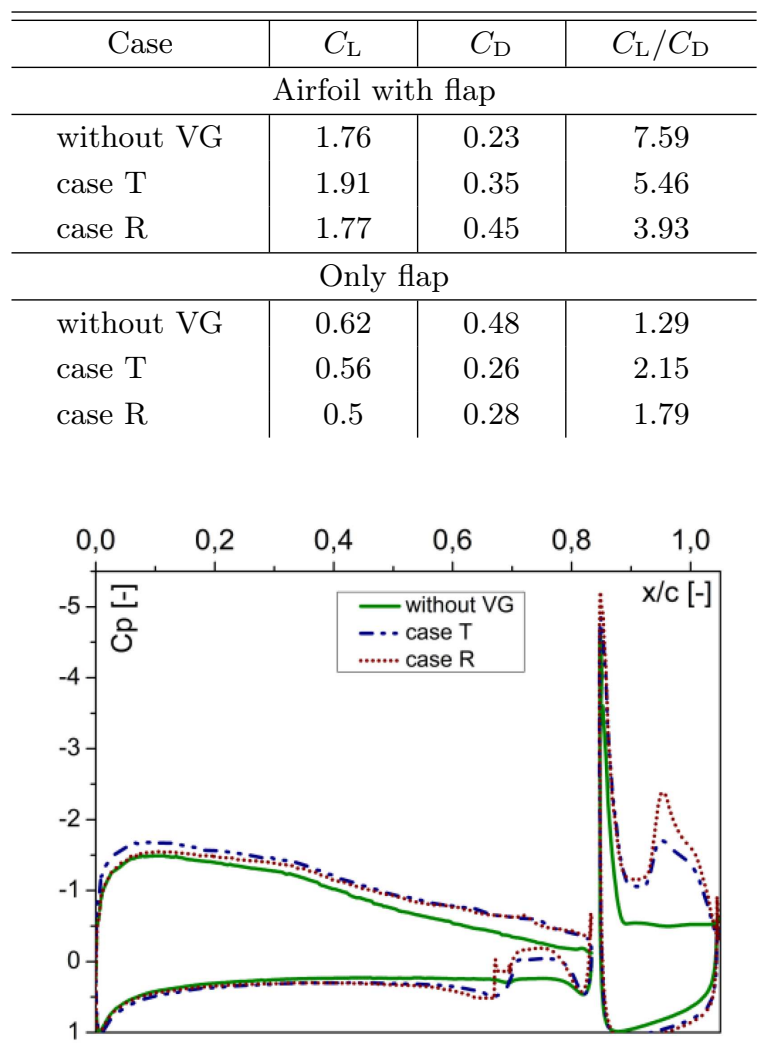

Fig. 4. Variation of pressure coefficient $C_{\mathrm{p}}$.

Examining the pressure distribution only on the airfoil, it can be seen that each generator causes a similar response, i.e., a slight increase of $C_{\mathrm{p}}$ on the lower side and a decrease on the upper side of the profile, which corresponds to the rise of the lift force, with the T-generator having a slightly more beneficial effect (see Table I). On the flap, on the other hand, a stronger response is observed on its upper side, which results in a slight decrease in the lift in this case.

The fundamental objective of aerodynamic airfoil design is to achieve the highest possible lift/drag ratio. However, when analyzing the $C_{\mathrm{L}} / C_{\mathrm{D}}$ ratio, a favorable effect was obtained only for the T-type generator. It can also be seen from the data that the flap plays an important role in the global value of this parameter. Therefore, the remaining part of this paper deals with the flow of this element only.

Figure 5a shows the magnitude velocity fields in the form of pathlines for the base configuration at a flap deflection of $\delta=55^{\circ}$. The velocity fields show that the flow is separated over the complete length of the flap resulting in a drastic loss in lift efficiency. The flow-separated area near the airfoil lower side trailing edge is also seen. Figure $5 \mathrm{~b}$ and $\mathrm{c}$ visualizes velocity fields for the configurations with triangle/rectangle shapes of VG, which in general shows a more favorable view of the flow compared
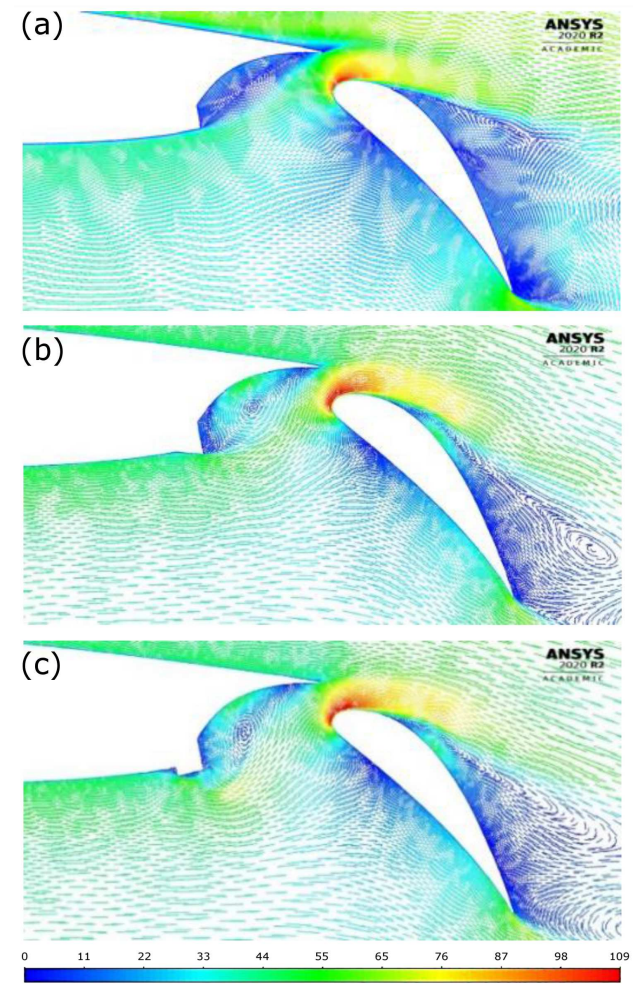

Fig. 5. The pathlines colored by Velocity Magnitude $\mathrm{W}[\mathrm{m} / \mathrm{s}]$ : (a) without $\mathrm{VG}$, (b) case $\mathrm{T}$, (c) case $\mathrm{R}$.

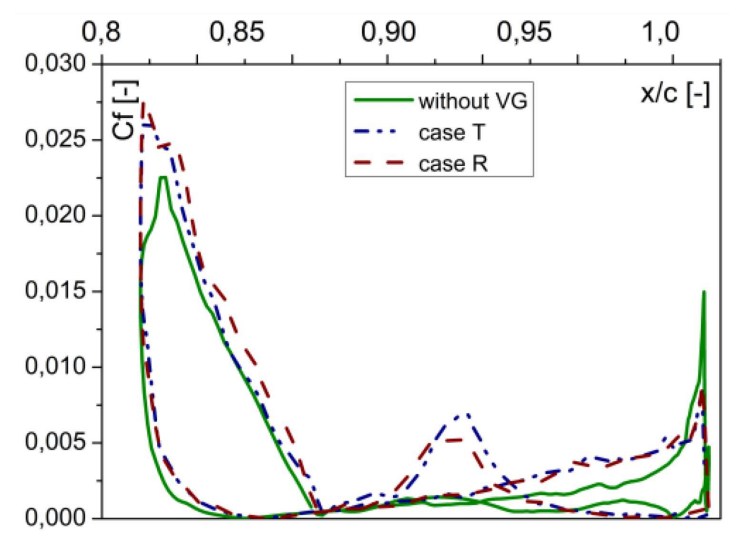

Fig. 6. Variation of skin friction coefficient $C_{\mathrm{f}}$.

to the baseline configuration. These pictures confirm also less deviated flow on the upper side and the delay of flow separation on the flap. The pictures of mean velocity fields, i.e., a shift in separation and the narrower aerodynamic wake behind the flap for VG configurations in comparison to the case without VG, give a visible increase in the lift coefficient.

To better show the pressure effects on the flap, the distribution of skin friction coefficient $C_{\mathrm{f}}$ on their upper surface is observed. From the graph in Fig. 6, a slight improvement can be seen for the configurations with vortex generators in the delay of flow separation. The effect of the presence of VG is 
not only a little delayed separation but also the increase of the flow turbulisation and adhesion of the boundary layer above $x=0.9 c$, which is responsible for a significant decrease in the $C_{\mathrm{D}}$ coefficient. As a result, for the T-generator, a nearly $70 \%$ increase of $C_{\mathrm{L}} / C_{\mathrm{D}}$ ratio is observed.

\section{Conclusions}

In this paper, aerodynamic characteristics of NLF-MOD22B airfoil have been studied using ANSYS Fluent by the SST $k-\omega$ turbulence model. Validation of the $2 \mathrm{D}$ model gives results comparable to the experiment performed by D.P. Jansen [2]. The obtained results indicate the delay of the position of flow separation for both analyzed configurations (the R- and T-generators) in comparison to the base case. But, only for the case with the T-generator, the aerodynamic factors are visibly better than for the basic case.

\section{Acknowledgments}

This work was supported by the Ministry of Science and Higher Education of Poland, No. BS-PB1-100-3011/2021/P, and PL-Grid Infrastructure.

\section{References}

[1] X.-K. Li, W. Liu, T.-J. Zhang, PP.-M. Wang, X.-D. Wang, Energies 12, 4583 (2019).

[2] D.P. Jansen, M.Sc. Thesis, Delft University of Technology, 2012.

[3] A. Dróżdż, P. Niegodajew, M. Romańczyk, V. Sokolenko, W. Elsner, Exp. Therm. Fluid Sci. 121, 110291 (2021).

[4] T.K. Barlas, G.A.M. van Kuik, Prog. Aerosp. Sci. 46, 1 (2010).

[5] S.J. Johnson, J.P. Baker, C.P. Van Dam, D. Berg, Wind Energy 13, 239 (2010).

[6] J.C. Lin, Prog. Aerosp. Sci. 38, 389 (2002).

[7] H. Wang, B. Zhang, Q. Qiu, X. Xu, Energy 118, 1210 (2017).

[8] J. Lin, in: Proc. 30th Fluid Dyn. Conf., 1999, p. 3404. 